\title{
MONITORING OF DEFORMATION OF THE FOGUANG TEMPLE'S EAST MAIN HALL UNDER THE CONCEPT OF PREVENTIVE CONSERVATION
}

\author{
YUMIN LI, RONG ZHANG \\ Culture Heritage Conservation Center of Beijing Guowenyan CO.,LTD \\ Beijing, China-37318215@qq.com
}

Commission II, WG II/8

KEY WORDS: Preventive Conservation, Monitoring of Deformation, The East Main Hall

\begin{abstract}
:
The East Main Hall of Foguang Temple is an immensely precious Tang Dynasty wooden building in China. Therefore, great caution must be exercised in the timely restoration and disease prevention of the heritage building. High-precision monitoring is carried out for timely detection of abnormalities and analysis of causes, so that reasonable measures are adopted to reduce or eliminate the risks faced by the hall in a timely manner and achieve preventive protection. Based on the description and purpose of preventive protection, this paper introduces in detail the technical route of monitoring of the East Main Hall and the analysis results of some monitoring data under the concept of preventive protection.
\end{abstract}

\section{PREVENTIVE PROTECTION AND MONITORING OF ANCIENT ARCHITECTURE}

Preventive protection chiefly refers to a host of measures that prevent, reduce or slow the damage of heritage. The purpose is to prevent repair and unnecessary maintenance under extreme emergency situations.

Unlike the rescue and protection of buildings, the preventive protection of ancient architecture stresses the information collection, precision survey, value assessment and risk assessment to determine the risks faced by ancient architecture. The law of change in damage to the heritage structure is analyzed through regular inspection and systematic monitoring. Measures such as disaster prevention, routine maintenance, and scientific management can reduce or eliminate risks in a timely manner, thereby preventing blind conservation projects. Monitoring is the basic means and indispensable aspect of preventive protection. It is the most fundamental and reliable method to find the extent and speed of damage to ancient architecture.

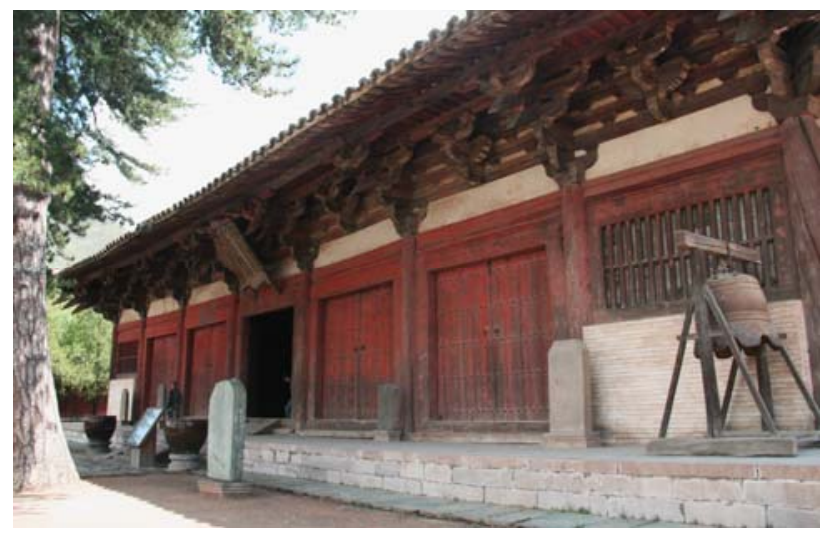

Fig.1 The East Main Hall of Foguang Temple

\section{MEASUREMENT OF THE EAST MAIN HALL IN 2005}

The East Main Hall of Foguang Temple is the only extant Tang Dynasty wooden structure in China. The temple preserves the clay sculptures, murals and inscriptions and a majority of wooden components from the Tang Dynasty. Called "the top treasure of Chinese ancient architecture" by Mr. Liang Sicheng, it has a special significance in the architectural culture area in China and even in East Asia as a whole. Therefore, the heritage preservation departments at all levels in China set great store by the preservation of the East Main Hall by conducting extensive investigations and research. In 2005, the project team conducted on-site survey, material inspection and digital recording, including scanning the East Main Hall using leading-edge 3D laser scanning equipment at that time. the team obtained accurate 3D information and carried out special studies such as measurement, research and disease damage analysis of large timber structures based on the 3D point cloud modeling. These data and analysis results provided a comparison basis for the subsequent survey and measurement, but due to constraints at that time, the data obtained in 2005 had the following weaknesses:

\section{Accuracy of 3D data}

The measurement was made using the highest precision Leica 3D laser scanning system, but the current mainstream equipment in the industry has certain improvement in terms of data acquisition accuracy and point density.

2. Coordinate system of 3D data

No long-term stable measurement reference points at the time of measurement in 2005 were established to provide a unified coordinate system for subsequent measurements.

\section{Completeness of 3D data}

Only the ground station was set up when scanning the exposed horizontal wood in 2005. The data points on the upper part of the beam were scarce and relatively incomplete. 


\section{DEFORMATION MONITORING OF THE EAST MAIN HALL}

In 2018, the project for monitoring deformation of the architecture in the East Main Hall was carried out. The East Main Hall was measured again and compared to the 3D data obtained in 2005 , in order to judge the change to the positions of column grid and the beam frame of the hall as well as the degree of change during the 13 years. At the same time, the project team set the measurement reference point, established control network, and accurately measured the observation nails placed on the column and beam frame to provide a precise unified measurement coordinate system for subsequent monitoring. On this basis, it is possible to judge whether the bottom of the column settles, whether the column cap is deviated, and whether the deflection of the beam frame is abnormal. Therefore, the technical route combining highprecision single-point measurement method and spatial 3D laser scanning technology was adopted. Using electronic level, highprecision prism-free total station and 3D laser scanner, the team measured the East Main Hall during the four hottest and coldest periods in two years. Based on the information on climate and environmental factors and others acquired during the same period, an overall analysis was carried out to explore the possible trend of deformation and the relationship between deformation and environment, and to provide technical standards for the continuous monitoring of the East Main Hall.
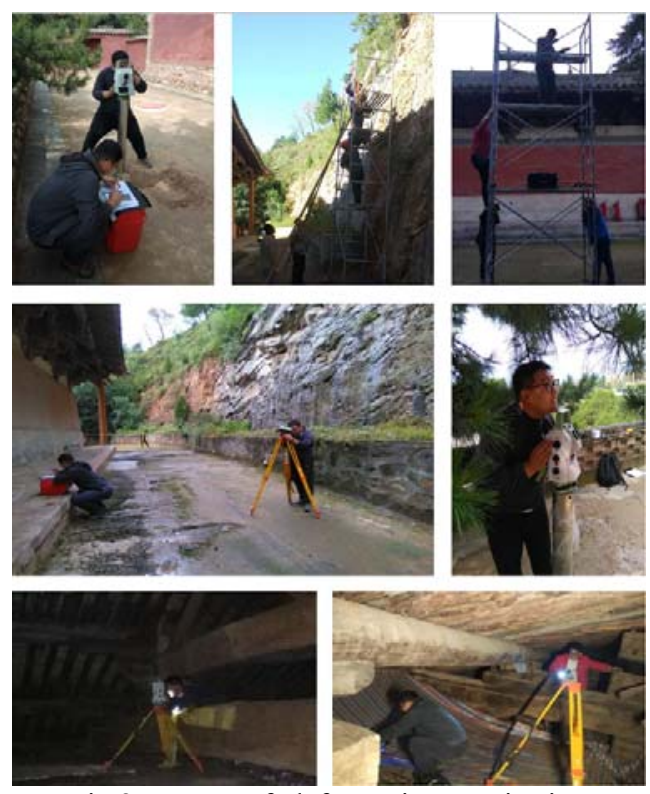

Fig.2 Process of deformation monitoring

\subsection{Control measurement}

The control network is the basis of the deformation monitoring. The control network includes the plane control network and the elevation control network. It adopts the first-order level and first-order horizontal displacement monitoring for the deformation monitoring under the engineering measurement specifications. According to the surrounding terrain of the East Main Hall, it is proposed to use precision wire as the plane control network. The plane control network comprises four wire points, which are located at the outer corners of the hall, forming a closed wire. The leveling network acts as an elevation control network. The monitoring reference points are measured according to the requirements of the Code for Deformation Measurement of Building and Structure.

\subsection{Monitoring point measurement}

Through research and comparison, it is determined that monitoring points are embedded at the bottom and top of the columns, the outside and the center of the crescent beam, and both sides and the center of beam frame and purlins of the East Main Hall. Displacement monitoring of the monitoring points is divided into two parts: plane displacement monitoring and settlement displacement monitoring.

3.2.1 Calculation of error in plane displacement monitoring After the calculation of the plane displacement monitoring, the station is set on the stable ground for observation, and the rootmean-square deviation (RMSD) is about $1 \mathrm{~mm}$ (see formula 1).

$$
m_{P}= \pm \sqrt{m_{s}{ }^{2}+\left(\frac{m_{\beta}}{\rho} * s\right)^{2}}
$$

where

$\mathrm{ms}=$ total station ranging error $(2 \mathrm{~mm})$

$\mathrm{m} \beta=$ total station angle measurement error $\left(0.5^{\prime \prime}\right)$

$\rho=$ operational constant (206265)

$\mathrm{S}=$ horizontal distance

The average is obtained from the readings in 4 observations, and the error in point measurement $m_{p}$ is

$$
m_{P}{ }^{s}= \pm \frac{2}{\sqrt{4}}= \pm 1 \mathrm{~mm}
$$

For the monitoring points with better observation conditions, the intersection measurement method is used. The error can be accurate within $0.1 \mathrm{~mm}$, see formula 2 ;

$$
m_{P}=M= \pm \frac{m_{p} * \sqrt{s_{a}^{2}+s_{b}^{2}}}{\rho^{*} \sin \gamma}
$$

where

$\mathrm{m}_{\beta}=$ total station angle measurement error $\left(0.5^{\prime \prime}\right)$

$\mathrm{S}_{\mathrm{a}}=$ The horizontal distance from the observation point $\mathrm{P}$ to the observation station a

$\mathrm{S} b=$ The horizontal distance from the observation point $\mathrm{P}$ to the observation station $b$

$\gamma=$ The Angle between the observation point $\mathrm{P}$ and the plane of station $a$ and $b$

$\rho=$ operational constant $\rho \quad(206265)$

It is hard to control error in some monitoring points in the horizontal wood in the ceiling. As the space in this area is small, and the many structures are mutually blocked, it is not suitable for intersection measurement. In addition, the instrument placed on the wood square grid will cause a slight vibration of the grid if it runs, increasing the measurement error. Therefore, the error in the observation points in the horizontal wood consists of the following: $0.05 \mathrm{~mm}$ RMSD for total station, $0.8 \mathrm{~mm}$ RMSD for intersection measurement (formula 3), RMSD for the station (formula 4), and cumulative error for the station-by-station progress (formula 5). After calculation, the RMSD in the final observation point is about $3 \mathrm{~mm}$.

$$
\begin{gathered}
M= \pm \frac{\sqrt{m_{a}^{2}+m_{b}^{2}}}{\sin \gamma} \\
m_{W}= \pm \sqrt{\left( \pm \frac{M}{\sqrt{4}}\right)^{2}+\frac{m_{r}^{2}}{4}} \\
m_{g}^{r}= \pm \sqrt{5} \& m_{W A}
\end{gathered}
$$


3.2.2 Calculation of error in settlement displacement monitoring

In the settlement displacement monitoring, the station is set up on the stable ground for observation, and the elevation is measured using the total station triangular elevation. The RMSD for the elevation is about $1.5 \mathrm{~mm}$ (see formula 6 and formula 7).

$$
\begin{aligned}
& = \pm \sqrt{\sin ^{2} a^{*} M_{L}{ }^{2}+\cos ^{2} a^{*} L^{2} * \frac{m_{a}{ }^{2}}{\rho^{2}}} \\
& m_{\mathrm{H} p}= \pm \sqrt{m_{H p}{ }^{2}+m_{P H p}{ }^{2}}
\end{aligned}
$$

where

$$
\begin{aligned}
& \mathrm{a}=\text { The observation angle }\left(\leqslant 45^{\circ}\right) \\
& \mathrm{L}=\text { The observation slant distance }(\leqslant 20 \mathrm{~m}) \\
& \mathrm{m}_{\mathrm{L}}=\text { total station ranging error }(2 \mathrm{~mm}) \\
& \mathrm{m}_{\mathrm{a}}=\text { total station angle measurement error }\left(0.5^{\prime \prime}\right) \\
& \rho=\text { operational constant }(206265)
\end{aligned}
$$

The measurement error of the horizontal wood also needs to take account of the cumulative error of the station-by-station progress, as shown in formula 8 . The RMSD of elevation in the final observation point is some $3 \mathrm{~mm}$.

$$
m_{H i}- \pm \sqrt{n} * m_{H p}{ }^{v}
$$

The above measurement process and error analysis results achieve the minimum measurement errors possible under the current technical conditions.

At present, the measurement of the first summer at the measurement points is completed, and the second stage is ongoing.

\subsection{Comparison of 3D data}

On the basis of the precision control network, the 3D scanning equipment is used to obtain the complete $3 \mathrm{D}$ information of the interior and exterior of the hall and the exposed beams and horizontal wood. 173 scanning measurements of the stations have been made.

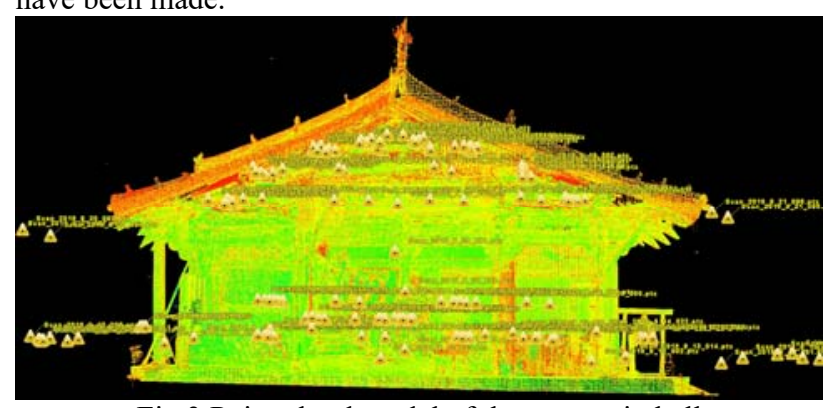

Fig.3 Point cloud model of the east main hall

The multi-station point cloud forms a complete $3 \mathrm{D}$ point cloud model through data collation. The scanning error of the point cloud is $0.3 \mathrm{~mm}$.

In the measurements in 2005 , the surface of the column base stone in front of the north seam of the outer room was used as the 0.00 elevation of the East Main Hall. This survey also uses this point as the 0.00 elevation (all elevation values in this article are the relative measurement values based on this reference point). According to the data analysis in 2005, the plane measurements of the column cap and base were first carried out. Based on the 3D point cloud data, 3D laser scanning point cloud slices about $50 \mathrm{~mm}$ thick were cut at an elevation of $500 \mathrm{~mm}$ (front peripheral column, base of hypostyle column), $1460 \mathrm{~mm}$ (base of posterior inner column), and $4750 \mathrm{~mm}$ (column cap). Each column slice is fitted to a circle, and the center-to-center gap is measured to obtain the width and depth dimensions at the height of each layer. Compared to the 2005 data, it is possible to find the changes to the direction of width and depth of the column network in the hall during the 13 years.

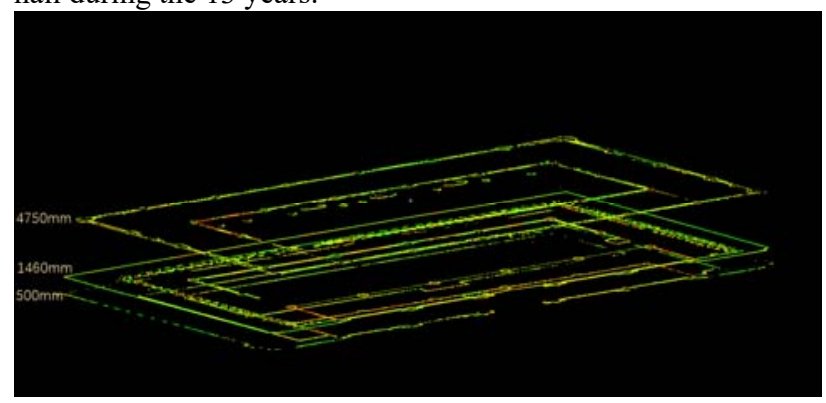

Fig.4 3 layers slice of point cloud

Excluding the errors caused by different measuring equipment and staff at the two measurements, and the inevitable measurement error, it is found that the trend of change to each column is basically the same in the direction of width, and the changes in the rear peripheral column and the posterior inner column are greater than that of other columns; in the direction of depth, the outermost columns in the first row see great changes, resulting in a great change in the depth.

The working group measured the deviation of the column cap and compared it to the data in 2005. From the numerical point of view, this column continues to expand southward and westward on the basis of the value in 2006, which may cause the growing westward deviation of the column cap in the south. The reason for the increase requires further study. Further, the eastern deviation of the southwest corner column is reduced compared to that in 2006, which is also a prominent change. We also measured the elevation of the column cap, and compared it with the value in 2006. There is a significant reduction in the front inner groove column in the north gable (see table 1 and fig 5). Therefore, based on the current monitoring values, the columns at the south and north corners of the front eaves see great changes, which is one of the focuses for our subsequent monitoring and analysis.

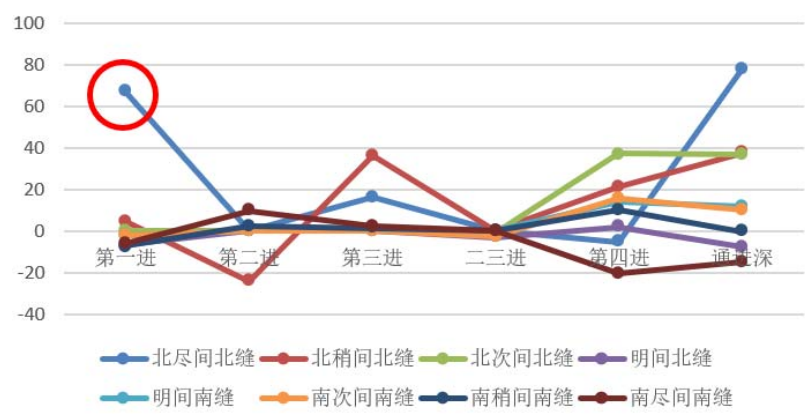

Fig.5 Coordinate of the top center of the column is abnormal. 


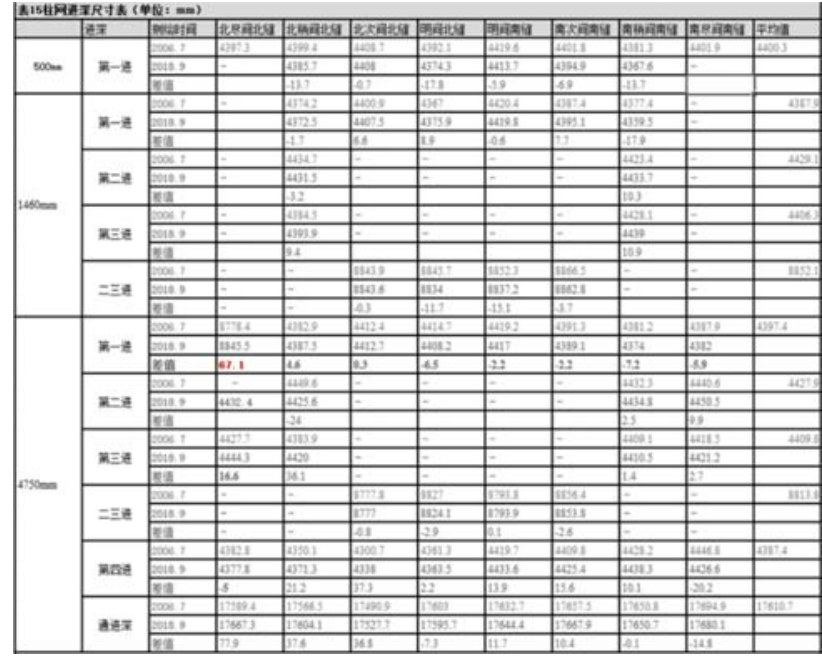

Table 1. Center distances from the top of the columns

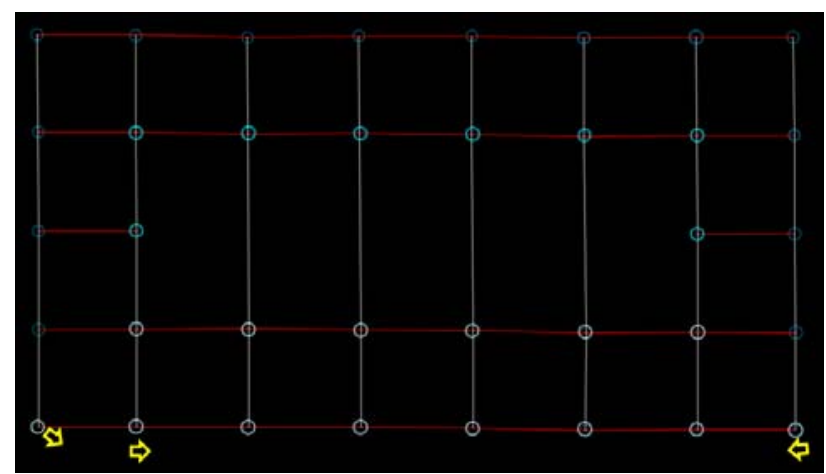

Fig.6 The positions of the column with abnormal data

\section{SUMMARY}

The East Main Hall of The Foguang Temple is one of the most important extant ancient architecture in China. It is of huge value in terms of spatial layout, building structure and processing of components. Great caution must be exercised in the timely protection and repair of the hall. Therefore, it is necessary to use state-of-the-art technology for continuous measurement, analyse the overall and local changes to the building structure, and provide basis for the development of scientific, reasonable protection measures in conjunction with relevant technical standards, with a view to achieving preventive protection. This is the purpose of heritage monitoring. It is necessary to make accurate and long-term monitoring of built heritage, and make regular recording of data on the status. Moreover, based on environmental monitoring, material process analysis and other aspects, coupled with the comprehensive analysis of the causes, scope and degree of the changes in architectural structure, we judge whether this change is a sound self-adjustment or severe structural deformation; and whether it is a stable state formed over a long time, or an active progressing disease.

This deformation monitoring takes into account the overall deformation and local slight deformation, and the error in each aspect of the measurement is strictly controlled. It aims to reduce errors as much as possible and improve the monitoring accuracy under current technical conditions. This measurement sets stable reference points to provide a unified coordinate system for subsequent measurement of various types. The data currently obtained are only raw data of long-term monitoring, but it has been found that the local structure of the building has data anomalies. By way of subsequent long-term monitoring, more comprehensive, detailed measurement data and analysis results can be obtained, thereby providing a reliable basis for the protection and preservation of the East Main Hall.

\section{REFERENCES}

Rong.Zhang., Xian.Lei., Qi.Wang., Ning.Lv., Shuai.Wang., 2018. A Study on Contruction Histroy of Main Hall of Foguangsi(Fo Kuang Ssu). Architectural History, 41(1), pp. 3152 .

Chengjun.Bai., Xu.Han., Cong.Wu., 2013. The Exploration of Architectural Heritage Deformation Monitoring in the Thought of Preventive Conservation. J.Xi'an Univ. of Arch.\& Tech, 32(2), pp. 54-58.

Hao.Chang.,2018. Deformation Monitoring System Design for Timberwork Ancient Buildings. He Bei University. 